\title{
Infecciones de Catéter Venoso Central y Lock Terapia en Pacientes Oncológicos
}

\author{
PAOLA KABALAN B. ${ }^{1}$, NATALIE RODRÍGUEZ Z. ${ }^{2}$, \\ JUAN TORDECILLA C. ${ }^{2}$, FANNY SEPÚLVEDA R. ${ }^{3}$ \\ 1. Pediatra, Becada Hemato-oncología pediátrica. Hospital Roberto del Río, Universidad de Chile. \\ 2. Pediatra Hemato-oncólogo. Hospital Roberto del Río, Universidad de Chile. \\ 3. Enfermera Unidad Oncología. Hospital Roberto del Río.
}

\begin{abstract}
Infections of the Central Line and Lock Therapy in Pediatric Oncological Children

Introduction: The use of a permanent Central Venous Catheter (CL) in oncologic patients allows a secure and rapid access. Infection is an important risk. "Lock therapy" (LT) consists of the application of a high concentration of antibiotics in the lumen of the catheter, for the purpose of eradicating bacteria. Objective: To describe the use of LT associated with the systemic use of antibiotics in CL infections occurring among pediatric oncological patients. Methods: A descriptive, restrospective review of charts of children seen at the Oncology Unit, Roberto del Rio Children's Hospital, 2005 to 2007. Evaluated parameters included culture and sentitivity studies, removal of CL and mortality. Results: There were 11 episodes of infection associated to $\mathrm{CL}$ in 8 patients, most frequently associated to Staphylococcus coagulase-negative. The CL was preserved in 7 patients. There was no mortality associated to infection. Conclusion: For this group, LT and systemic antibiotics preserved the CL in most patients. Randomized prospective studies are needed in a larger population to support these findings.
\end{abstract}

(Key words: Lock Therapy, Central Venous Catheter, oncologic, children, infection).

Rev Chil Pediatr 2010; 81 (5): 425-431

\section{RESUMEN}

Introducción: El uso de catéter venoso central permanente (CVC) en pacientes oncológicos aporta un acceso venoso seguro y expedito. Sin embargo, la infección es una de las principales complicaciones asociadas a su uso. La "lock terapia" es una técnica que consiste en la aplicación en el lúmen del catéter de una solución con alta concentración de antibiótico, con el objetivo de erradicar las bacterias presentes en el endolumen. Objetivo: Describir el uso de "lock terapia" asociada a antibióticos sistémicos en infecciones relacionadas a CVC en pacientes pediátricos oncológicos. Métodos: Estudio descriptivo, retrospectivo, donde se analizaron las

Trabajo recibido el 29 de septiembre de 2009, devuelto para corregir el 22 de febrero de 2010, segunda versión el 25 de mayo de 2010, tercera versión el 07 de septiembre de 2010, aceptado para publicación el 13 de septiembre de 2010.

Correspondencia a:

Paola Kabalan B.

E-mail: pkabalan@gmail.com 
infecciones asociadas a CVC que fueron tratadas con lock terapia y antibióticos sistémicos en la Unidad de Oncología del Hospital de Niños Roberto del Río entre los años 2005 y 2007. Los parámetros estudiados fueron: cultivo y antibiograma; retiro del CVC y mortalidad. Resultados: Se estudiaron 11 episodios de infección asociada a CVC en 8 pacientes. El microorganismo aislado con mayor frecuencia fue Staphylococcus coagulasa negativo. Se logró conservar el CVC en 7 pacientes. No hubo mortalidad asociada a infección. Conclusiones: En el grupo estudiado, el uso asociado de lock terapia y antibióticos sistémicos permitió conservar el CVC en la mayoría de los casos. Se requieren estudios prospectivos randomizados con un mayor número de pacientes que permitan corroborar estos hallazgos.

(Palabras clave: Lock terapia, catéter venoso central, paciente oncológico).

Rev Chil Pediatr 2010; 81 (5): 425-431

\section{Introducción}

Los niños con patología oncológica son un grupo de pacientes que con frecuencia utilizan un catéter venoso central (CVC) permanente, con el fin de contar con un acceso venoso expedito y seguro que permita la administración de medicamentos, hemoderivados, nutrición parenteral y la obtención de muestras para exámenes de laboratorio, mejorando de esta manera la calidad de su atención. Las infecciones asociadas al uso de este tipo de CVC en estos pacientes son un problema relevante, tanto por su morbimortalidad, como por el costo que implica para los servicios de salud y por los riesgos a los que son sometidos los pacientes con el eventual retiro o la reimplantación de estos $^{1-4}$.

El último informe de vigilancia epidemiológica de infecciones intrahospitalarias del Ministerio de Salud, año 2007, muestra una tasa de 1,82 infecciones por 1000 días de CVC transitorios en pacientes pediátricos, siendo Staphylococcus aureus y Staphylococcus coagulasa negativo los microorganismos más frecuentemente aislados con un $28,3 \%$ cada uno. Con respecto a las Infecciones del torrente sanguíneo en pacientes pediátricos en tratamiento con drogas antineoplásicas, la tasa por 1000 días de neutropenia asciende a 3,67 donde Klebsiella pneumoniae es el agente aislado con más frecuencia 5 .

Las principales fuentes de colonización de los CVC son la piel y la conexión externa. Además algunas bacterias tienen la capacidad de producir biofilm (membrana viscosa de po- lisacárido), capaz de proteger a los microorganismos, lo que dificulta la erradicación del agente infeccioso en estos dispositivos ${ }^{1,3,6}$.

El uso de antibióticos sistémicos no siempre logra la esterilización del CVC permanente, por lo tanto, el paciente sigue expuesto a complicaciones o recurrencia de la infección. La forma más efectiva de erradicar el agente infeccioso sería el retiro del CVC. Debido a la dificultad de obtener un acceso venoso y el riesgo que implica un procedimiento quirúrgico en los pacientes oncológicos, se ha intentado preservar los catéteres permanentes usando terapia antibiótica sistémica, combinada o no con el sistema de lock terapia ${ }^{1-7,8}$.

La lock terapia es una técnica que consiste en la aplicación en el lúmen del catéter de una solución con alta concentración de antimicrobiano, por varias horas, con el objetivo de erradicar las bacterias presentes en el endolumen y así prolongar la vida útil del catéter, reduciendo la morbimortalidad y los costos asociados a una infección relacionada a $\mathrm{CVC}^{4,9-11}$.

Existen publicaciones internacionales discordantes en relación a los resultados y beneficios de la lock terapia, que se basan en recomendaciones de expertos y series clínicas con escaso número de pacientes, faltando estudios randomizados que avalen su uso. Según algunos grupos, la tasa de erradicación de los microorganismos es de aproximadamente un $80 \%$ con terapia sistémica asociada a lock terapia, en cambio, con terapia antibiótica sistémica exclusiva, disminuye la tasa de erradicación a un $70 \%$, presentando además frecuentes recidivas. En Chile, actualmente no existen 
datos publicados en este grupo de pacientes pediátricos, que validen o no el uso de lock terapia $^{4,7,9-11}$.

Nuestro objetivo fue describir la experiencia del uso de lock terapia asociada a tratamiento antibiótico sistémico en pacientes pediátricos oncológicos que presentaban infección relacionada a $\mathrm{CVC}$.

\section{Pacientes y Métodos}

\section{Selección de pacientes}

Se trata de un estudio descriptivo, retrospectivo, realizado en la Unidad de HematoOncología del Hospital de Niños Roberto del Río en el período comprendido entre enero 2005 y diciembre 2007. En este período, se instalaron 59 catéteres con reservorio subcutáneo y 2 catéteres de larga duración tipo Hickmann, dependiendo de las características de cada paciente.

Se incluyeron pacientes portadores de CVC permanente, menores de 20 años, que presentaron infección relacionada a catéter diagnosticada si cumplían al menos 1 requisito de los que aparecen definidos en el consenso nacional sobre infecciones asociadas a catéteres vasculares centrales publicado el año $2003^{12}$ :

- Hemocultivos cuantitativos simultáneos a través del catéter y por venopunción con una razón $\geq 4: 1$ (sangre por catéter $v s$ sangre periférica).

- Tiempo diferencial hasta detectarse crecimiento bacteriano, de al menos 2 horas entre el hemocultivo obtenido por catéter y el hemocultivo periférico.

Fueron excluidos aquellos pacientes con una infección relacionada a CVC en quienes fue necesario el retiro de éste por: gravedad clínica (trombosis séptica, endocarditis infecciosa, osteomielitis), tipo de microorganismo (Candida, Staphylococcus coagulasa positivo, Corinebacterium jk) y/o tunelitis o infección del bolsillo subcutáneo.

Todos los pacientes que presentaron una infección relacionada a CVC, recibieron terapia antibiótica sistémica asociada a lock terapia, de acuerdo al protocolo nacional del manejo de neutropenia febril del grupo PINDA (Programa Infantil Nacional de Drogas Antineoplásicas del Ministerio de Salud de Chile). Por tratarse de un grupo de pacientes en control permanente en nuestra Unidad, no se realizó hemocultivo en forma rutinaria al finalizar el tratamiento con lock terapia ${ }^{13}$.

Los padres o tutores de los pacientes firmaron un consentimiento informado previo al inicio de la quimioterapia que describe las complicaciones infecciosas y el manejo de ellas.

Durante el período de análisis de los casos no existía vigilancia de infecciones en los pacientes con CVC permanente, por lo que no se cuenta con un grupo de control histórico.

\section{Técnica de lock terapia}

Los antibióticos utilizados en lock terapia deben ser estables en el lumen del catéter durante todo el tiempo que permanecen en él, deben ser compatibles con el tipo de catéter utilizado y con el resto de los componentes de la lock terapia (heparina, suero fisiológico u otros antibióticos) ${ }^{4}$. Se preparan concentraciones estándares del antibiótico a utilizar según la recomendación de la Sociedad Chilena de Infectología, como aparecen en la tabla 1. Se aplica sobre el lumen afectado durante 12 horas nocturnas, entre 7 a 14 días $^{12}$. Una variedad de soluciones han sido evaluadas; una de las más estudiadas es la mezcla de vancomicina

\section{Tabla 1. Diluciones de antibióticos en lock terapia (Recomendación de la Sociedad Chilena de Infectología. Consenso $2003^{12}$ )}

\begin{tabular}{|c|c|}
\hline Antibiótico & Preparación \\
\hline Cefazolina $(10 \mathrm{mg} / \mathrm{ml})$ & $\begin{array}{l}\text { Cefazolina } 1 \mathrm{ml}+\text { heparina } \\
0,5 \mathrm{ml}+\text { suero fisiológico } \\
0,5 \mathrm{ml}\end{array}$ \\
\hline Gentamicina ( 4 mg/ml) & $\begin{array}{l}\text { Gentamicina } 0,5 \mathrm{ml}+\text { hepari- } \\
\text { na } 0,5 \mathrm{ml}+\text { suero fisiológico } \\
1 \mathrm{ml}\end{array}$ \\
\hline Cefazolina-gentamicina & $\begin{array}{l}\text { Gentamicina } 0,5 \mathrm{ml}+\text { cefa- } \\
\text { zolina } 1 \mathrm{ml}+\text { heparina } 0,5 \mathrm{ml}\end{array}$ \\
\hline Vancomicina (5 mg/ml) & $\begin{array}{l}\text { Vancomicina } 1 \mathrm{ml}+\text { heparina } \\
0,5 \mathrm{ml}+\text { suero fisiológico } \\
0,5 \mathrm{ml}\end{array}$ \\
\hline Vancomicina-gentamicina & $\begin{array}{l}\text { Vancomicina } 1 \mathrm{ml}+\text { gentami- } \\
\text { cina } 0,5 \mathrm{ml}+\text { heparina } 0,5 \mathrm{ml}\end{array}$ \\
\hline
\end{tabular}


y heparina, considerando la incidencia de infecciones relacionadas a CVC por Staphylococcus coagulasa negativo. Considerando la presencia de fibronectina, fibrinógeno y fibrina en el lumen del CVC, el uso de heparina aumentaría la eficacia del antibiótico utilizado. Se puede adicionar antimicrobianos con actividad contra microorganismos gram negativos, como gentamicina o amikacina. La elección del antimicrobiano guarda directa relación con el microorganismo aislado causante de la infección ${ }^{4-14}$.

\section{Variables estudiadas}

En este grupo se registró las características del paciente en relación a edad, diagnóstico oncológico de base, tipo de CVC permanente, presencia o no de neutropenia asociada al episodio de infección relacionado a $\mathrm{CVC}$, microorganismo aislado, tratamiento antibiótico sistémico y lock terapia utilizada. Se evaluó si fue necesario o no retirar el catéter, el motivo del retiro y la evolución post tratamiento en relación con recurrencia de la infección, complicaciones mecánicas del CVC, y aparición de infección sistémica.

\section{Análisis estadístico}

Para el análisis se utilizó estadística descriptiva debido a la naturaleza del estudio donde se analizaron y describieron los datos recolectados sin realizar inferencias estadísticas.

\section{Resultados}

En el período estudiado se evaluaron 11 episodios, que se presentaron en 8 pacientes, con una proporción de 1,3 episodios por paciente. Las edades fluctuaron entre 1 y 17 años, con una mediana de 4 años. Todos los pacientes estaban en tratamiento con quimioterapia activa al momento del diagnóstico de la infección asociada a CVC. Se evidenció neutropenia severa en 6 episodios. Los otros 5 tenían recuento de neutrófilos normal y todos eran portadores de CVC con reservorio subcutáneo.

El diagnóstico oncológico más frecuente en los pacientes estudiados fue leucemia linfática aguda (tabla 2).
De acuerdo al Consenso de la Sociedad Chilena de Infectología del año 2003, en 3 episodios se hizo el diagnóstico con hemocultivos cuantitativos simultáneos y en 2 episodios fue a través de tiempo diferencial entre los hemocultivos. En el resto de los eventos, fueron ambas técnicas las que confirmaron el diagnóstico

Los microorganismos aislados en los 11 episodios se detallan en la tabla 3 , destacando como agente más frecuente el Staphylococcus coagulasa negativo. El resto de microorganismos fueron todos gram negativos. Con respecto al hallazgo de Pseusomona no aeruginosa en uno de los pacientes, no se retiró el CVC ya que la tipificación de este microorganosmo se recibió en forma diferida, logrado una buena respuesta al tratamiento empírico con amikacina.

Todos los pacientes recibieron terapia antibiótica sistémica asociada a lock terapia. Hubo 7 episodios que se manejaron sólo con terapia

Tabla 2. Diagnóstico oncológico en los pacientes estudiados

\begin{tabular}{|lc|}
\hline Diagnóstico & $\begin{array}{c}\text { n de } \\
\text { episodios }\end{array}$ \\
\hline Leucemia linfática aguda de alto riesgo & 4 \\
Neuroblastoma etapa IV & 3 \\
\hline Leucemia mieloide aguda & 2 \\
Leucemia linfática aguda recaída & 1 \\
Linfoma de Burkitt & 1 \\
\hline
\end{tabular}

Tabla 3. Microorganismos aislados

\begin{tabular}{|lc|}
\hline Microorganismos & $\begin{array}{c}\text { n de } \\
\text { episodios }\end{array}$ \\
\hline Staphylococcus coagulasa negativo & 6 \\
Klebsiella pneumoniae & 1 \\
Enterobacter aerogenes & 1 \\
Acinetobacter no baumanii & 1 \\
Escherichia coli & 1 \\
Pseudomonas no aeruginosa* & 1 \\
\hline *No se retira CVC por buena evolución clínica y necesidad de \\
acceso venoso.
\end{tabular}


Tabla 4. Lock terapia utilizada

\begin{tabular}{|cllcc|}
\hline Episodio & Microorganismo & Lock terapia & Tiempo lock terapia & $\begin{array}{c}\text { Tiempo total de terapia } \\
\text { antibiótica e.v }\end{array}$ \\
\hline 1 & S. coagulasa negativo & Vancomicina & 7 días & 7 días \\
\hline 2 & S. coagulasa negativo & Vancomicina & 7 días & 13 días \\
\hline 3 & S. coagulasa negativo & Vancomicina & 7 días & 5 días \\
\hline 4 & S. coagulasa negativo & Vancomicina & 14 días & 10 días \\
\hline 5 & S. coagulasa negativo & Vancomicina & 14 días & 10 días \\
\hline 6 & S. coagulasa negativo & Cefazolina & 12 días & 9 días \\
\hline 8 & Enterobacter aerogenes & Amikacina & 10 días & 10 días \\
\hline 9 & Acinetobacter no baumanii & Amikacina & 14 días & 10 días \\
10 & Escherichia coli & Amikacina & 14 días & 10 días \\
11 & Pseudiella pneumoniae & Gentamicina & 14 días & 14 días \\
\hline
\end{tabular}

S: Staphylococcus.

de primera línea (Cloxacilina $200 \mathrm{mg} / \mathrm{kg} /$ día, Cefotaxima $150 \mathrm{mg} / \mathrm{kg} /$ día y Amikacina 15 $\mathrm{mg} / \mathrm{kg} / \mathrm{día}$ ) y 4 en que se cambió Cloxacilina por Vancomicina $(40 \mathrm{mg} / \mathrm{kg} /$ día $)$. Destaca la adición de Metronizadol (30 mg/kg/día) en 2 episodios en que se objetivó foco abdominal. Ceftazidima (100 mg/kg/día) e Imipenem (40 $\mathrm{mg} / \mathrm{kg} /$ día) fueron utilizados sólo en 1 paciente. Ningún paciente utilizó antifúngico ya que la infección por hongos se consideró criterio de exclusión e indicación de retiro de CVC desde el diagnóstico del episodio. En relación con la lock terapia utilizada, destaca el uso de vancomicina en 5 de 6 episodios causados por Staphylococcus coagulasa negativa. En los microorganismos gram negativos, la alternativa más utilizada fue amikacina. El detalle de los tratamientos indicados en cada episodio se observa en la tabla 4.

$\mathrm{Al}$ analizar la evolución clínica, sólo hubo 1 episodio que requirió manejo en unidad de cuidados intensivos en sus primeras 48 horas de evolución debido a compromiso hemodinámico inicial. No fue necesario el uso de drogas vasoactivas ni ventilación mecánica. Un paciente presentó un segundo episodio de infección relacionada a CVC a igual microorganismo (Klebsiella pneumoniae), lo que motivó el retiro del CVC. No hubo mortalidad en el grupo estudiado.
En 7 pacientes se logró conservar el CVC permanente.

\section{Discusión}

Se han desarrollado diversas estrategias para evitar el riesgo de bacteriemias derivadas de la infección de CVC permanente, siendo una de ellas el uso de antibióticos profilácticos en bajas concentraciones. Existe un metaanálisis publicado el año 2006 que demuestra la utilidad del uso de lock terapia con vancomicina en la prevención de infecciones relacionadas a CVC, pero sólo se recomienda su uso en cierto grupo de pacientes (portadores de "intestino corto" o en hemodiálisis). Existen trabajos en pacientes oncológicos pediátricos donde se recomienda el uso de lock terapia en el tratamiento de infecciones relacionadas a CVC no complicadas, sobretodo si el microorganismo aislado es el Staphylococcus coagulasa negativo $^{10,14,15}$.

Han sido estudiadas una gran variedad de soluciones para lock terapia, siendo la más utilizada la mezcla de vancomicina y heparina, considerando que el patógeno más frecuentemente aislado en bacteriemia relacionada a CVC es el Staphylococcus coagulasa negativo, como lo confirmamos en el estudio de nues- 
tros pacientes y como lo describe la literatura, además se observaron resultados favorables en la mayoría de nuestros pacientes tratados con lock terapia ${ }^{4,10,16}$.

El tratamiento con lock terapia de las bacteriemias del torrente sanguíneo asociadas a CVC permanente, está basado principalmente en series clínicas, recomendaciones de expertos, guías clínicas y un reducido número de estudios de casos-controles. Por lo que aun se debe definir el tiempo de duración de la lock terapia. Las series describen desde infusión rápida de 1 hora, diariamente, hasta instilaciones mensuales en pacientes portadores de catéteres con reservorio subcutáneo que se atienden en forma ambulatoria. Con respecto a la concentración del antibiótico, la mayoría de las series utiliza una concentración 10 a 20 veces la CIM. Sin embargo, hay grupos que recomiendan 100-5 000 veces su valor. Nosotros utilizamos la recomendación de la Sociedad Chilena de Infectología en que se utiliza la lock terapia por 12 horas, por un período total de tratamiento entre 7 y 14 días $^{4,10,16}$.

No hay evidencia suficiente para asegurar que el uso de lock terapia no genere el desarrollo de cepas resistentes, pero la práctica clínica parece no demostrarlo, ya que se logra la erradicación del microorganismo en un alto porcentaje asociado a terapia sistémica ${ }^{4,7,10,12,14,16}$.

Otros grupos han descrito experiencias satisfactorias con la instilación de etanol en el lumen de los catéteres infectados, sin uso de antibiótico, logrando la erradicación del microorganismo aislado en el $97 \%$ de los episodios. Otro fármaco estudiado es la taurolidina, derivado del aminoácido taurina que posee una amplia acción bactericida y fungicida. A diferencia de los antibióticos, no selecciona cepas resistentes. En algunos estudios se ha utilizado como profilaxis primaria o acompañada de antibióticos en tratamiento de infecciones relacionadas a CVC. En nuestra unidad, no se han desarrollado este tipo de técnicas, y se requieren más estudios en pacientes pediátricos para instaurar su uso ${ }^{16}$.

Como se ha mencionado antes, existen trabajos que no son comparables entre si y que no permiten sacar conclusiones definitivas y esto hace que el uso de lock terapia aparezca todavía como controversial. Sin embargo, quisimos describir la experiencia del uso de esta terapia en pacientes pediátricos oncológicos, porque aunque se trata de un número reducido de episodios, que no permite obtener conclusiones definitivas, en nuestra experiencia ha demostrado que permite la mantención del $\mathrm{CVC}$, disminuyendo el riesgo de morbimortalidad que involucra el retiro y la reinstalación de un CVC.

Aunque aún hay muchas preguntas no resueltas en la literatura en relación a este tipo de tratamiento (tiempo de duración de lock terapia, concentración óptima de antibiótico o qué microorganismos pueden ser tratados en forma segura), creemos que el uso de lock terapia asociada a terapia antibiótica sistémica es una alternativa válida y segura en el manejo de las infecciones relacionadas a CVC, si las condiciones clínicas del paciente lo permiten. Sin embargo, se deben realizar otros estudios con mayor número de casos para avalar su uso generalizado.

\section{Agradecimientos}

Al equipo de enfermería oncológica del Hospital Roberto del Río por su labor permanente e incondicional apoyo para realizar este trabajo.

\section{Referencias}

1.- Ferretti $G$, Mandalà M, Di Cosimo S, Moro C, Curigliano G, Barni S: Catheter related bloodstream infections, part I: Pathogenesis, diagnosis and management Infections in Oncology. Cancer Control 2002; 9: 513 22.

2.- Munro F, Gillet P, Shaw M, Thomas A, Mackinlay G: Totally implantable central venous access devices for paediatric oncology patients. Medical and Pediatric Oncology 1999; 33: 377-81.

3.- Marco del Pont J, Paganini H, Debbag R, et al: Consenso nacional. Riesgo de infección en el paciente oncológico. Arch Argentina de Pediatría 2003; 101 (4): 270-94.

4.- Bestul M, Van den Bussche L: Antibiotic lock technique: Review of the literature. Pharmacotherapy 2005; 25 (2): 
211-27.

5.- Bustamante R, Espinola $V$ : Informe de vigilancia epidemiológica de infecciones intrahospitalarias. Unidad de Infecciones Intrahospitalarias. Departamento de Calidad en Salud Subsecretaría de Redes Asistenciales MINSAL, Chile. 2007.

6.- Mermel L, Farr B, Sherertz R, et al: Guidelines for the Management of Intravascular Catheter-Related Infections. Clin Infect Dis 2001; 32: 1249-72.

7.- Oncu S, Oncu S, Ozrtuk B, Kurt I, Sakarya S: Elimination of intraluminal colonization by antibiotic lock in catheters. Tohoku J Exp Med 2004; 203: 1-8.

8.- García P, Payá E, Olivares R, Cotera A, Rodríguez $J$, Sanz M: Diagnóstico de las infecciones asociadas a catéteres vasculares centrales. Rev Chil Infect 2003; 20 (1): 41-50.

9.- Carratala J: The antibiotic-lock technique for therapy of 'highly needed' infected catheters. European Society of Clinical Microbiology and Infectious Diseases 2002; 8: 282-9.

10.- Fortun J, Grill F, Martin-Dávila P, et al: Treatment of long-term intravascular cathether-related bacteraemia with antibiotic-lock therapy. J Antimicrob Chemother 2006; 58: 816-21.

11.- Berrington A, Gould K: Use of antibiotic lock to treat colonized central venous catheters. J Antimicrob Chemother 2001; 48: 597-603.

12.- Fica $C$ : Consenso nacional sobre infecciones asociadas a catéteres vasculares centrales. Rev Chil Infect 2003; 20 (1): 39-40.

13.- Santolaya M, Rabagliati R, Bidart T, et al: Consenso Manejo racional del paciente con cáncer, neutropenia y fiebre. Rev Chil Infect 2005; 22 (supl 2): S79-S113.

14.- Toltiz P: Antibiotic lock technique to reduce central venous catheter-related bacteremia. Pediatr Infect Dis J 2006; 25 (5): 449-50.

15.- Safdar N, Maki D: Use of Vancomycin-Containing Lock or Flush Solutions for Prevention of Bloodstream Infection Associated with Central Venous Access Devices: A Meta-Analysis of Prospective, Randomized Trials. Clin Infect Dis 2006; 43: 478-84.

16.- Simon A, Bode U, Beutel K: Diagnosis and treatment of catheter-related infections in paediatric oncology. An update. Clin Microbiol Infect 2006; 12: 606-20. 